\title{
WEIGHTED AND VECTOR-VALUED INEQUALITIES FOR POTENTIAL OPERATORS
}

\author{
FRANCISCO J. RUIZ BLASCO AND JOSÉ L. TORREA HERNANDEZ
}

\begin{abstract}
In this paper we develop some aspect of a general theory parallel to the Calderón-Zygmund theory for operator valued kernels, where the operators considered map functions defined on $R^{n}$ into functions defined on $R_{+}^{n+1}=R^{n} \times[0, \infty)$.

In particular, we apply the obtained results to get vector-valued inequalities for the Poisson integral and fractional integrals. Some weighted norm inequalities are also considered for fractional integrals.
\end{abstract}

1. Introduction. The main objective of this paper is to find weighted and vector-valued inequalities for some operators mapping functions defined on $R^{n}$ into functions on $R_{+}^{n+1}=R^{n} \times[0, \infty)$.

We now mention some of the concrete operators we shall consider and the corresponding inequalities:

(I) The Poisson integral:

$$
\mathcal{P} f(x, t)=\int_{R^{n}} f(y) P(x-y, t) d y \quad\left(x \in R^{n}, t \geq 0\right),
$$

where

$$
P(x, t)=c_{n} t\left(|x|^{2}+t^{2}\right)^{-(n+1) / 2}
$$

is the Poisson kernel.

(II) The maximal operator $\mathcal{M}$ introduced by Fefferman and Stein [1]:

$$
\mathcal{M} f(x, t)=\sup _{Q}\left\{\frac{1}{|Q|} \int_{Q}|f(y)| d y\right\} \quad\left(x \in R^{n}, t \geq 0\right)
$$

where the supremum is taken over the cubes $Q$ in $R^{n}$ centered at $x$ with sides parallel to the axes and has side length at least $t$.

(III) The generalized fractional integral of order $\alpha(0<\alpha<n)$ :

$$
T_{\alpha} f(x, t)=c_{n} \int_{R^{n}} \frac{f(y)}{(|x-y|+t)^{n-\alpha}} d y \quad\left(x \in R^{n}, t \geq 0\right) .
$$

(IV) The maximal operator $\mathcal{M}_{\alpha}(0 \leq \alpha<n)$, which controls (in a sense that will be made precise later) the fractional integral of order $\alpha$ :

$$
\mathcal{M}_{\alpha} f(x, t)=\sup _{Q}\left\{\frac{1}{|Q|^{1-\alpha / n}} \int_{Q}|f(y)| d y\right\} \quad\left(x \in R^{n}, t \geq 0\right),
$$

Received by the editors April 4, 1985.

1980 Mathematics Subject Classification. Primary 42B20, 42B25.

Key words and phrases. Poisson integral, Carleson measures, fractional integral, vectorvalued inequalities, weighted norm inequalities. 
where the supremum is taken over the cubes $Q$ in $R^{n}$ centered at $x$ with sides parallel to the axes and has side length at least $t$ (observe that $\mathcal{M}_{0}=\mathcal{M}$ ).

The vector-valued inequalities that we shall obtain will be of the type

$$
\left\{\int_{R_{+}^{n+1}}\left(\sum_{j}\left|T f_{j}\right|^{r}\right)^{q / r} d \mu\right\}^{1 / q} \leq C\left\{\int_{R^{n}}\left(\sum_{j}\left|f_{j}\right|^{r}\right)^{p / r} d x\right\}^{1 / p}
$$

and

$$
\left(\mu\left\{(x, t) \in R_{+}^{n+1}: \sum_{j}\left|T f_{j}\right|^{r}>\lambda^{r}\right\}\right)^{1 / q} \leq \frac{C}{\lambda} \int_{R^{n}}\left(\sum_{j}\left|f_{j}\right|^{r}\right)^{1 / r} d x
$$

for $\mu$ a generalized Carleson measure, i.e., $\mu(\tilde{Q}) \leq C|Q|^{\delta}$, where $\tilde{Q}$ denotes the cube in $R_{+}^{n+1}$ with the cube $Q$ as its basis, $|Q|$ denotes the Lebesgue measure of $Q$ in $R^{n}, \delta \geq 1$, and $C$ is a constant.

In the case $T=\mathcal{M}$ of $T=P$ the inequalities (1) and $\left(1^{\prime}\right)$ are valid respectively for $1<p=q<\infty$ and $q=1$, with $\delta=1$ in both cases.

In the case $T=\mathcal{M}_{\alpha}$ or $T=T_{\alpha}$ and $\delta=s(1-\alpha / n) \geq 1,(1)$ and $\left(1^{\prime}\right)$ are valid respectively for $1 / p=\alpha / n+s(1-\alpha / n) / q, s<q<\infty$ and $q=s$.

The fact that inequalities (1) and $\left(1^{\prime}\right)$ are true for $P$ and $\mathcal{M}$ could be established after the work of J. L. Rubio de Francia [5], in which some relation between vectorvalued inequalities and weighted norm inequalities are proved, and the papers of $F$. J. Ruiz and J. L. Torrea [7, 8], where some kind of weighted norm inequalities for the operators $P$ and $\mathcal{M}$ are established.

Although the operator $P$ is controlled pointwise by $\mathcal{M}$ and, therefore, would be sufficient to obtain the vector-valued inequalities $(1)$ and $\left(1^{\prime}\right)$ for $\mathcal{M}$, we shall develop a general technique parallel to the Calderón-Zygmund theory for vectorvalued functions (see J. L. Rubio de Francia, F. J. Ruiz and J. L. Torrea [6]) which will allow us to consider the two operators $P$ and $\mathcal{M}$ as singular integrals of a general type and to get, indistinctly, $(1)$ and $\left(1^{\prime}\right)$ for $P$ and $\mathcal{M}$.

The organization of this paper is as follows: in $\S 2$ we introduce all notations and we state the main results; the proofs are given in $\S 3$; in $\S 4$ we apply the above results to obtain, in particular, (1) and $\left(1^{\prime}\right)$ for $\mathcal{P}, \mathcal{M}, T_{\alpha}$ and $\mathcal{M}_{\alpha}$. Finally, in $\S 5$, we prove some weighted norm inequalities for $\mathcal{M}_{\alpha}$.

Throughout this paper the letter $C$ will be used to denote a positive constant, not necessarily the same at each occurrence.

2. Main theorems. The letters $A$ and $B$ will denote arbitrary Banach spaces, $\mathcal{L}(A, B)$ will be the set of bounded linear operators from $A$ to $B$.

Let $\mu$ be a positive measure on $R_{+}^{n+1}$ and $\omega$ a positive measurable function in $R^{n}$. Given $0 \leq \alpha<n$ and $1<p, q<\infty$ we shall say that the pair $(\mu, \omega)$ satisfies condition $C_{q, p, \alpha}\left(\right.$ or $\left.(\mu, \omega) \in C_{q, p, \alpha}\right)$ if for any cube $Q$ in $R^{n}$

$$
\frac{\mu(\tilde{Q})^{1 / q}}{|Q|^{(1 / p)-(\alpha / n)}}\left(\frac{1}{|Q|} \int_{Q} \omega(x)^{-p^{\prime} / p} d x\right)^{1 / p^{\prime}} \leq C
$$

$\left(p^{\prime}\right.$ always means the conjugate exponent of $p$, i.e. $\left.(p-1)\left(p^{\prime}-1\right)=1\right)$. 

if

We shall say that $(\mu, \omega)$ satisfies condition $C_{q, 1, \alpha}$ for $1 \leq q<\infty$ and $0 \leq \alpha<n$

$$
\sup _{Q \ni x} \frac{\mu(\tilde{Q})^{1 / q}}{|Q|^{1-\alpha / n}} \leq C \omega(x) \quad \text { a.e. } x .
$$

The cases $\alpha=0$ and $1 \leq p=q<\infty$ have already been considered in [7]; we shall simply write $C_{p}$ in this case.

If $\omega \equiv 1$, the above conditions are of the type $\mu(\tilde{Q}) \leq C|Q|^{\delta}$, i.e. $\mu$ is a generalized Carleson measure of order $\delta$.

We shall denote by $L_{A}^{\dot{p}}\left(R^{n} ; d x\right), 1 \leq p \leq \infty$, the Bochner-Lebesgue space of $A$-valued strong measurable functions $f$ such that $\int_{R^{n}}\|f(x)\|_{A}^{p} d x<\infty$. Analogously we define $L_{A}^{p}\left(R^{n} ; \omega(x) d x\right)$ or $L_{B}^{p}\left(R_{+}^{n+1} ; d \mu\right)$. Sometimes, we shall write in an abridged form $L_{A}^{p}(d x), L_{A}^{p}(d \omega)$ or $L_{B}^{p}(d \mu)$.

Now we state the main theorems.

THEOREM 1. Let $A, B$ be Banach spaces, $0<\alpha<n$ and $s$ such that $0<1 / s \leq$ $1-\alpha / n$. Let $T$ be a bounded linear operator from $L_{A}^{n / \alpha}(d x)$ into $L_{B}^{\infty}(d \mu)$, where $\mu$ is a generalized Carleson measure of order $s(1-\alpha / n)$.

Suppose that there exists a $\mathcal{L}(A, B)$-valued function $K$ in

$$
R^{n} \times R^{n} \times R^{+} \backslash\left\{(x, x, t): x \in R^{n}, t \geq 0\right\}
$$

such that:

(a) For any pair $(x, t) \in R_{+}^{n+1}, y \rightarrow K(x, y, t)$ is locally integrable and if $f$ is in $L_{A}^{n / \alpha}(d x)$ with compact support contained in a cube $Q$,

$$
T f(x, t)=\int_{R^{n}} K(x, y, t) f(y) d y \quad \text { for }(x, t) \notin \tilde{Q} .
$$

(b)

$$
\int_{\left|x-y^{\prime}\right|+t>2\left|y-y^{\prime}\right|}\left\|K(x, y, t)-K\left(x, y^{\prime}, t\right)\right\|_{\mathcal{L}(A, B)}^{s} d \mu(x, t) \leq C \quad \text { for } y, y^{\prime} \in R^{n}
$$

Then:

(i) $T$ maps $L_{A}^{p}(d x)$ into $L_{B}^{q}(d \mu)$ for $1 / p=1-\theta(1-\alpha / n), 1 / q=(1-\theta) / s$, $0<\theta \leq 1$ (i.e. $1 / p=\alpha / n+s(1-\alpha / n) / q, s<q<\infty)$.

(ii) $T$ maps $L_{A}^{1}(d x)$ into weak- $L_{B}^{s}(d \mu)$, i.e.

$$
\mu\left\{(x, t) \in R_{+}^{n+1}:\|T f(x, t)\|_{B}>\lambda\right\} \leq \frac{C}{\lambda^{s}}\left(\int_{R^{n}}\|f(x)\|_{A} d x\right)^{s} .
$$

REMARK. The last theorem remains true when $\alpha=0$ (i.e. $n / \alpha=\infty$ ), but in this case we are interested in the following result.

THEOREM 2. Let $A, B$ be Banach spaces and $T$ be a bounded linear operator from $L_{A}^{\infty}(\omega(x) d x)$ into $L_{B}^{\infty}(d \nu)$ for any pair $(\nu, \omega)$ in $C_{1}$.

Suppose that there exists a $\mathcal{L}(A, B)$-valued function $K$ in

$$
R^{n} \times R^{n} \times R^{+} \backslash\left\{(x, x, t): x \in R^{n}, t \geq 0\right\}
$$


such that:

(a) For any pair $(x, t) \in R_{+}^{n+1}, y \rightarrow K(x, y, t)$ is locally integrable and if $f$ is in $L_{A}^{\infty}(d x)$ with compact support contained in a cube $Q$,

$$
T f(x, t)=\int_{R^{n}} K(x, y, t) f(y) d y \quad \text { for }(x, t) \notin \tilde{Q} .
$$

(b)

$$
\left\|K(x, y, t)-K\left(x, y^{\prime}, t\right)\right\|_{\mathcal{L}(A, B)} \leq C \frac{\left|y-y^{\prime}\right|}{\left(\left|x-y^{\prime}\right|+t\right)^{n+1}}
$$

for $\left|x-y^{\prime}\right|+t>2\left|y-y^{\prime}\right|$.

Then, the following vector-valued inequalities hold for any Carleson measure $\mu$ on $R_{+}^{n+1}$ :

(i) For $1<p, q<\infty$

$$
\left\|\left(\sum_{j}\left\|T f_{j}\right\|_{B}^{q}\right)^{1 / q}\right\|_{L^{p}(d \mu)} \leq C\left\|\left(\sum_{j}\left\|f_{j}\right\|_{A}^{q}\right)^{1 / q}\right\|_{L^{p}(d x)} .
$$

(ii) For $1<q<\infty$

$$
\mu\left(\left\{(x, t) \in R_{+}^{n+1}: \sum_{j}\left\|T f_{j}(x, t)\right\|_{B}^{q}>\lambda^{q}\right\}\right) \leq \frac{C}{\lambda} \int_{R^{n}}\left(\sum_{j}\left\|f_{j}(x)\right\|_{A}^{q}\right)^{1 / q} d x
$$

In order to prove Theorem 2 we shall use the following

THEOREM 3. If $T$ is an operator verifying the hypothesis in Theorem 2, then:

(i) $T$ maps $L_{A}^{p}(\omega(x) d x)$ into $L_{B}^{p}(d \nu)$ for $1<p<\infty$ and $(\nu, \omega)$ in $C_{1}$.

(ii) $T$ maps $L_{A}^{1}(\omega(x) d x)$ into weak- $L_{B}^{1}(d \nu)$ for any $(\nu, \omega)$ in $C_{1}$.

3. Proofs of the main theorems. We shall first prove Theorem 3. It is enough to prove (ii) from which, by applying Marcinkiewicz's interpolation theorem, we obtain (i).

We shall denote $C_{1}(\nu, \omega)$ as the infimum of constants $C$ satisfying the condition $C_{1}$ for the pair $(\nu, \omega)$.

Let $f$ be a function in $L_{A}^{1} \cap L_{A}^{\infty}\left(R^{n} ; \omega(x) d x\right)$. Let $\lambda$ be a positive number and consider the dyadic cubes in $R^{n}$ such that

$$
\lambda<\frac{1}{|Q|} \int_{Q}\|f(x)\|_{A} d x
$$

Our goal is to prove

$$
\nu\left(\left\{(x, t) \in R_{+}^{n+1}:\|T f(x, t)\|_{B}>\lambda\right\}\right) \leq \frac{C}{\lambda} \int_{R^{n}}\|f(x)\|_{A} \omega(x) d x .
$$

We consider two possibilities: If

$$
\nu\left(R_{+}^{n+1}\right) \leq \frac{C_{1}(\nu, \omega)}{\lambda} \int_{R^{n}}\|f(x)\|_{A} \omega(x) d x
$$

then (4) is trivial. 
In the other case, the existence of dyadic maximal cubes verifying (3) is guaranteed by the following inequality:

$$
\nu(\tilde{Q}) \leq \frac{\nu(\tilde{Q})}{\lambda} \frac{1}{|Q|} \int_{Q}\|f(x)\|_{A} d x \leq \frac{C_{1}(\nu, \omega)}{\lambda} \int_{Q}\|f(x)\|_{A} \omega(x) d x
$$

where in the second inequality the hypothesis on $(\nu, \omega)$ is used.

As usual these dyadic maximal cubes, say $Q_{k}$, verify

$$
\lambda<\frac{1}{\left|Q_{k}\right|} \int_{Q_{k}}\|f(x)\|_{A} d x \leq 2^{n} \lambda
$$

and

$$
\|f(x)\|_{A} \leq \lambda \text { a.e. } x \notin \bigcup_{k} Q_{k}
$$

We shall introduce the following notation: $Q^{*}$ will be the cube with the same center as $Q$ but with side length two times the side length of $Q$. $\Omega$ will be the set union of the dyadic maximal cubes $\left(\Omega=\bigcup_{k} Q_{k}\right)$. Similarly, we shall denote $\Omega^{*}=\bigcup_{k} Q_{k}^{*}, \tilde{\Omega}=\bigcup_{k} \tilde{Q}_{k}$ and $\tilde{\Omega}^{*}=\bigcup_{k} \tilde{Q}_{k}^{*}$.

. We decompose $f=g+b$, where $g(x)=f(x)$ for $x \notin \Omega, g(x)=\left|Q_{k}\right|^{-1} \int_{Q_{k}} f(y) d y$ for $x \in Q_{k}$, and

$$
b(x)=f(x)-g(x)=\sum_{k}\left(f(x)-\frac{1}{\left|Q_{k}\right|} \int_{Q_{k}} f(y) d y\right) \chi_{Q_{k}}(x)=\sum_{k} b_{k}(x) .
$$

In order to prove (4) we use

$$
\|T g\|_{L_{B}^{\infty}(d \nu)} \leq C\|g\|_{L_{A}^{\infty}(d \omega)} \leq 2^{n} C \lambda
$$

to obtain

$$
\begin{aligned}
& \quad \nu\left(\left\{(x, t) \in R_{+}^{n+1}:\|T f(x, t)\|_{B}>2^{n+1} C \lambda\right\}\right) \\
& \quad \leq \nu\left(\left\{(x, t) \in R_{+}^{n+1}:\|T b(x, t)\|_{B}>2^{n} C \lambda\right\}\right)
\end{aligned}
$$

and, therefore, it is enough to show that

$$
\nu\left(\left\{(x, t) \in R_{+}^{n+1}:\|T b(x, t)\|_{B}>\lambda\right\}\right) \leq \frac{C}{\lambda} \int_{R^{n}}\|f(x)\|_{A} \omega(x) d x .
$$

We put

$$
\begin{aligned}
& \nu\left(\left\{(x, t) \in R_{+}^{n+1}:\|T b(x, t)\|_{B}>\lambda\right\}\right) \\
& \leq \nu\left(\tilde{\Omega}^{*}\right)+\nu\left(\left\{(x, t) \notin \tilde{\Omega}^{*}:\|T b(x, t)\|_{B}>\lambda\right\}\right) .
\end{aligned}
$$

Let us estimate the two members of this sum:

$$
\begin{aligned}
\nu\left(\tilde{\Omega}^{*}\right) & \leq \sum_{k} \nu\left(\tilde{Q}_{k}^{*}\right) \leq \sum_{k} \frac{\nu\left(\tilde{Q}_{k}^{*}\right)}{\lambda} \frac{1}{\left|Q_{k}\right|} \int_{Q_{k}}\|f(y)\|_{A} d y \\
& \leq 2^{n} C_{1}(\nu, \omega) \sum_{k} \frac{1}{\lambda} \int_{Q_{k}}\|f(y)\|_{A} \omega(y) d y \\
& \leq \frac{C}{\lambda} \int_{R^{n}}\|f(y)\|_{A} \omega(y) d y
\end{aligned}
$$


For the second member we apply hypothesis (a) to get

$$
\begin{aligned}
& \nu\left(\left\{(x, t) \notin \tilde{\Omega}^{*}:\|T b(x, t)\|_{B}>\lambda\right\}\right) \\
& \quad \leq \frac{1}{\lambda} \int_{\left(\tilde{\Omega}^{*}\right)^{c}}\|T b(x, t)\|_{B} d \nu \leq \frac{1}{\lambda} \sum_{k} \int_{\left(\tilde{\Omega}^{*}\right)^{c}}\left\|T b_{k}(x, t)\right\|_{B} d \nu \\
& \quad \leq \frac{1}{\lambda} \sum_{k} \int_{\left(\tilde{\Omega}^{*}\right)^{c}}\left(\int_{Q_{k}}\left\|K(x, y, t)-K\left(x, y_{k}, t\right)\right\|_{\mathcal{L}(A, B)}\left\|b_{k}(y)\right\|_{A} d y\right) d \nu
\end{aligned}
$$

where we have used that $\int_{Q_{k}} b_{k}=0$ and $y_{k}$ is the center of $Q_{k}$. By Fubini's theorem this is equal to

$$
\frac{1}{\lambda} \sum_{k} \int_{Q_{k}}\left\|b_{k}(y)\right\|_{A} \int_{\left(\tilde{\Omega}^{*}\right)^{c}}\left\|K(x, y, t)-K\left(x, y_{k}, t\right)\right\|_{\mathcal{L}(A, B)} d \nu(x, t) d y .
$$

Using hypothesis (b) this is less than

$$
\begin{aligned}
& \frac{C}{\lambda} \sum_{k} \int_{Q_{k}}\left\|b_{k}(y)\right\|_{A} \int_{\left(\tilde{\Omega}^{*}\right)^{c}} \frac{\left|y-y_{k}\right|}{\left(\left|x-y_{k}\right|+t\right)^{n+1}} d \nu(x, t) d y \\
& \quad \leq \frac{C}{\lambda} \sum_{k}\left(\int_{Q_{k}}\left\|b_{k}(y)\right\|_{A} d y\right) \cdot M_{k}=\frac{2 C}{\lambda} \sum_{k}\left(\int_{Q_{k}}\|f(y)\|_{A} d y\right) \cdot M_{k},
\end{aligned}
$$

where

$$
M_{k}=\underset{y \in Q_{k}}{\operatorname{ess} \sup _{\left(\tilde{\Omega}^{*}\right)^{c}}} \frac{\left|y-y_{k}\right|}{\left(\left|x-y_{k}\right|+t\right)^{n+1}} d \nu(x, t) .
$$

But for $y \in Q_{k}$ geometric considerations say that

$$
\begin{aligned}
& \int_{\left(\tilde{\Omega}^{*}\right)^{c}} \frac{\left|y-y_{k}\right|}{\left(\left|x-y_{k}\right|+t\right)^{n+1}} d \nu(x, t) \leq C\left|Q_{k}\right|^{1 / n} \sum_{j=1}^{\infty} \int_{A_{k}^{j}} \frac{d \nu(x, t)}{\left(\left|x-y_{k}\right|+t\right)^{n+1}} \\
& \leq \leq\left|Q_{k}\right|^{1 / n} \sum_{j=1}^{\infty} \int_{A_{k}^{j}} \frac{d \nu(x, t)}{\left(2^{j}\left|Q_{k}\right|^{1 / n}\right)^{n+1}} \\
& \leq C\left|Q_{k}\right|^{1 / n} \sum_{j} \frac{\nu\left(2^{j+1} Q_{k}\right)}{\left(2^{j}\left|Q_{k}\right|^{1 / n}\right)^{n+1}} \\
&=C \sum_{j=1}^{\infty} \frac{1}{2^{j}} \frac{\nu\left(2^{j+1} Q_{k}\right)}{\left|2^{j+1} Q_{k}\right|} \\
& \leq C \cdot C_{1}(\nu, \omega) \cdot \omega(x) \quad \text { a.e. } x \in Q_{k},
\end{aligned}
$$

where $A_{k}^{j}=\left\{(x, t) \in R_{+}^{n+1}: 2^{j+1}\left|Q_{k}\right|^{1 / n}>\left|x-y_{k}\right|+t>2^{j}\left|Q_{k}\right|^{1 / n}\right\}$ and $2^{j+1} Q_{k}$ denotes the cube in $R^{n}$ with the same center as $Q_{k}$ and side length $2^{j+1}$ times the length of $Q_{k}$. Therefore, we have $M_{k} \leq C \omega(x)$ a.e. $x \in Q_{k}$ and so

$$
\begin{aligned}
& \nu\left(\left\{(x, t) \notin \tilde{\Omega}^{*}:\|T b(x, t)\|_{B}>\lambda\right\}\right) \\
& \quad \leq \frac{C}{\lambda} \sum_{k} \int_{Q_{k}}\|f(y)\|_{A} \omega(y) d y \leq \frac{C}{\lambda} \int_{R^{n}}\|f(y)\|_{A} \omega(y) d y .
\end{aligned}
$$

This finishes the proof of Theorem 1 . 
REMARK. In the case $\omega(x) \equiv 1,(\mu, \omega) \in C_{1}$ if and only if $\mu$ is a Carleson measure. In this case, the first hypothesis on $T$ and condition (b) of Theorem 3 can be substituted by edly.

$\left(\mathrm{c}^{\prime}\right)$ For a fixed $p_{0}, 1<p_{0}<\infty, T \operatorname{maps} L_{A}^{p_{0}}\left(R^{n} ; d x\right)$ into $L_{B}^{p_{0}}\left(R_{+}^{n+1} ; d \mu\right)$ bound-

$\left(\mathrm{b}^{\prime}\right) \int_{\left|x-y^{\prime}\right|+t>2\left|y-y^{\prime}\right|}\left\|K(x, y, t)-K\left(x, y^{\prime}, t\right)\right\|_{\mathcal{L}(A, B)} d \mu(x, t) \leq C$ for $y, y^{\prime} \in R^{n}$ and then the next theorem can be proved.

THEOREM 4. If $T$ is an operator verifying $\left(\mathrm{b}^{\prime}\right)$ and $\left(\mathrm{c}^{\prime}\right)$ above and part (a) of Theorem 2, then

(i) $T$ maps $L_{A}^{p}\left(R^{n} ; d x\right)$ into $L_{B}^{p}\left(R_{+}^{n+1} ; d \mu\right)$ for $1<p \leq p_{0}$,

(ii) $T$ maps $L_{A}^{1}\left(R^{n} ; d x\right)$ into weak- $L_{B}^{1}\left(R_{+}^{n+1} ; d \mu\right)$.

The proof is as in Theorem 3 except the two following computations:

$$
\begin{aligned}
& \mu\left(\left\{(x, t) \in R_{+}^{n+1}:\|T g(x, t)\|_{B}>\lambda\right\}\right) \\
& \quad \leq \frac{1}{\lambda^{p_{0}}} \int_{R_{+}^{n+1}}\|T g(x, t)\|_{B}^{p_{0}} d \mu(x, t) \leq \frac{C}{\lambda^{p_{0}}} \int_{R^{n}}\|g(x)\|_{A}^{p_{0}} d x \\
& \quad \leq \frac{C}{\lambda} \int_{R^{n}}\|g(x)\|_{A} d x \leq \frac{C}{\lambda} \int_{R^{n}}\|f(x)\|_{A} d x
\end{aligned}
$$

and

$$
\begin{aligned}
& \sum_{k} \int_{Q_{k}}\left\|b_{k}(y)\right\|_{A} \int_{\left(\tilde{\Omega}^{*}\right)^{c}}\left\|K(x, y, t)-K\left(x, y_{k}, t\right)\right\|_{\mathcal{L}(A, B)} d \mu(x, t) d y \\
& \leq \sum_{k} C \int_{Q_{k}}\left\|b_{k}(y)\right\|_{A} d y \leq 2 C \sum_{k} \int_{Q_{k}}\|f(y)\|_{A} d y .
\end{aligned}
$$

PROOF OF THEOREM 2. Given an operator $T$ as in Theorem 2, we can define a new operator $\tilde{T}$ mapping $l^{s}(A)$-valued functions into $l^{s}(B)$-valued ones (where $s$ is fixed, $1<s<\infty)$ as

$$
\tilde{T}\left(f_{1}, f_{2}, \ldots, f_{j}, \ldots\right)=\left(T f_{1}, T f_{2}, \ldots, T f_{j}, \ldots\right) .
$$

By Theorem 3,T maps $L_{A}^{q}\left(R^{n} ; d x\right)$ into $L_{B}^{q}\left(R_{+}^{n+1} ; d \mu\right)(1<q<\infty)$; then it is clear that $\tilde{T}$ is bounded from $L_{l^{q}(A)}^{q}\left(R^{n} ; d x\right)$ to $L_{l_{(B)}^{q}}^{q}\left(R_{+}^{n+1} ; d \mu\right), 1<q<\infty$.

Moreover, $\tilde{T}$ is an operator like $T$, but with associated kernel $\tilde{K}(x, t)=K(x, t) \otimes$ Id, so that

$$
\|\tilde{K}(x, t)\|_{\mathcal{L}\left(l^{q}(A), l^{q}(B)\right)}=\|K(x, t)\|_{\mathcal{L}(A, B)} .
$$

Now, by Theorem 4, taking $l^{q}(A)$ and $l^{q}(B)$ as the Banach spaces and $q=p_{0}$, we obtain part (ii) of Theorem 2 and also part (i) with the restriction $1<p \leq q<\infty$.

To prove part (i) in the case $1<q<p<\infty$ we shall need the following

LEMMA. Let $u$ be a function in $L^{r}\left(R_{+}^{n+1} ; d \mu\right), 1<r \leq \infty$, and $\mu$ a Carleson measure. Consider the maximal function

$$
u^{*}(x)=\sup _{x \in Q} \frac{1}{|Q|} \int_{\tilde{Q}}|u(x, t)| d \mu(x, t) .
$$

Then

$$
\left\|u^{*}\right\|_{L^{r}\left(R^{n} ; d x\right)} \leq C\|u\|_{L^{r}\left(R_{+}^{n+1} ; d \mu\right)}
$$


Before proving the lemma we shall finish the proof of Theorem 2.

Let $r=p / q$ and make the following computation:

$$
\int_{R_{+}^{n+1}}\left(\sum_{j}\left\|T f_{j}\right\|_{B}^{q}\right)^{p / q} d \mu=\left(\int_{R_{+}^{n+1}}\left(\sum_{j}\left\|T f_{j}\right\|_{B}^{q}\right) u d \mu\right)^{r}
$$

where $u \geq 0, u \in L^{r^{\prime}}\left(R_{+}^{n+1} ; d \mu\right)$ and $\|u\|_{L^{r^{\prime}}(d \mu)} \leq 1$.

It is obvious that the pair $\left(u d \mu, u^{*}\right)$ satisfies condition $C_{1}$. Then by Theorem 3 the last member of $(7)$ is less than

$$
\begin{aligned}
\left(\int_{R^{n}} \sum_{j}\left\|f_{j}(x)\right\|_{A}^{q} u^{*}(x) d x\right)^{r} \cdot C & \leq C\left(\int_{R^{n}}\left(\sum_{j}\left\|f_{j}(x)\right\|_{A}^{q}\right)^{r} d x\right) \cdot\left\|u^{*}\right\|_{L^{r^{\prime}}(d x)}^{r} \\
& \leq C \int_{R^{n}}\left(\sum_{j}\left\|f_{j}(x)\right\|_{A}^{q}\right)^{p / q} d x
\end{aligned}
$$

where in the last inequality we have used the lemma. This concludes the proof of Theorem 2.

PROOF OF THE LEMMA. Observe that

$$
\left|u^{*}(x)\right| \leq \sup _{x \in Q} \frac{\mu(\tilde{Q})}{|Q|}\|u\|_{L^{\infty}(d \mu)} \leq C\|u\|_{L^{\infty}(d \mu)}
$$

that is,

$$
\left\|u^{*}\right\|_{L^{\infty}(d x)} \leq C\|u\|_{L^{\infty}(d \mu)}
$$

Then it is enough to prove

$$
\left|\left\{x \in R^{n}: u^{*}(x)>\alpha\right\}\right| \leq \frac{C}{\alpha} \int_{R_{+}^{n+1}}|u(x, t)| d \mu(x, t)
$$

since the rest follows by interpolation with the result for $r=\infty$.

But (8) can be done by a standard application of Besicovitch's covering lemma.

PROOF OF THEOREM 1. As in Theorem 3, it is enough to prove (ii). In order to do that we shall make a kind of "variable" Calderón-Zygmund decomposition (the idea goes back to [12 and 13]).

Our goal is to prove

$$
\mu\left(\left\{(x, t) \in R_{+}^{n+1}:\|T f(x, t)\|_{B}>\gamma\right\}\right) \leq \frac{C}{\gamma^{s}}\left(\int_{R^{n}}\|f(x)\|_{A} d x\right)^{s} .
$$

Given a function $f \in L_{A}^{1} \cap L_{A}^{n / \alpha}(d x)$, let $\lambda$ be any positive number; if $Q$ is a cube verifying (3), then it is obvious that $|Q|<\lambda^{-1} \int_{R^{n}}\|f(x)\|_{A} d x$ and this allows us to make all the constructions in the proof of Theorem 3, and in particular the decomposition $f=g+b$ corresponding to the number $\lambda$.

In order to obtain (9) we shall begin with

$$
\mu\left(\left\{(x, t) \in R_{+}^{n+1}:\|T b(x, t)\|_{B}>\gamma\right\}\right) .
$$

This is less than

$$
\mu\left(\tilde{\Omega}^{*}\right)+\mu\left(\left\{(x, t) \notin \tilde{\Omega}^{*}:\|T b(x, t)\|_{B}>\gamma\right\}\right) .
$$


The first member of this sum satisfies (we put $s_{0}=s(1-\alpha / n)$ )

$$
\begin{aligned}
\mu\left(\tilde{\Omega}^{*}\right) & \leq \sum_{k} \mu\left(\tilde{Q}_{k}^{*}\right) \leq \sum_{k} \frac{\mu\left(\tilde{Q}_{k}^{*}\right)}{\left|Q_{k}\right|^{s_{0}}} \cdot \frac{1}{\lambda^{s_{0}}}\left(\int_{Q_{k}}\|f(y)\|_{A} d y\right)^{s_{0}} \\
& \leq \frac{C}{\lambda^{s_{0}}}\left(\int_{R^{n}}\|f(y)\|_{A} d y\right)^{s_{0}},
\end{aligned}
$$

where the hypothesis on $\mu$ and the fact that $s_{0} \geq 1$ have been used in the last inequality.

On the other hand

$$
\begin{aligned}
\mu(\{(x, t) & \left.\left.\notin \tilde{\Omega}^{*}:\|T b(x, t)\|_{B}>\gamma\right\}\right)^{1 / s} \\
\leq & \frac{C}{\gamma}\left(\int_{\left(\tilde{\Omega}^{*}\right)^{c}}\|T b(x, t)\|_{B}^{s} d \mu(x, t)\right)^{1 / s} \\
\leq & \frac{C}{\gamma} \sum_{k}\left(\int_{\left(\tilde{Q}_{k}^{*}\right)^{c}}\left\|T b_{k}(x, t)\right\|_{B}^{s} d \mu(x, t)\right)^{1 / s} \\
\leq & \frac{C}{\gamma} \sum_{k}\left\{\int_{\left(\tilde{Q}_{k}^{*}\right)^{c}}\left(\int_{Q_{k}}\left\|K(x, y, t)-K\left(x, y_{k}, t\right)\right\|\left\|b_{k}(y)\right\| d y\right)^{s} d \mu(x, t)\right\}^{1 / s} \\
\leq & \frac{C}{\gamma} \sum_{k} \int_{Q_{k}}\left(\int_{\left(\tilde{Q}_{k}^{*}\right)^{c}}\left\|K(x, y, t)-K\left(x, y_{k}, t\right)\right\|^{s} d \mu(x, t)\right)^{1 / s}\left\|b_{k}(y)\right\| d y \\
\leq & \frac{C}{\gamma} \sum_{k} \int_{Q_{k}}\left\|b_{k}(y)\right\| d y \leq \frac{C}{\gamma} \int_{R^{n}}\|f(y)\|_{A} d y,
\end{aligned}
$$

where Minkowski's inequality for $d m(y)=\left\|b_{k}(y)\right\| d y$, the hypothesis on $K$, and the disjointness of $Q_{k}$ have been used.

The above estimates add up to get

$$
\begin{aligned}
& \mu\left(\left\{(x, t) \in R_{+}^{n+1}:\|T b(x, t)\|_{B}>\gamma\right\}\right) \\
& \quad \leq C\left\{\left(\frac{1}{\lambda} \int_{R^{n}}\|f(y)\|_{A} d y\right)^{s(1-\alpha / n)}+\left(\frac{1}{\gamma} \int_{R^{n}}\|f(y)\|_{A} d y\right)^{s}\right\} .
\end{aligned}
$$

In particular we can choose $\lambda$ such that

$$
\frac{1}{\lambda^{1-\alpha / n}}=\frac{1}{\gamma}\left(\int_{R^{n}}\|f(y)\|_{A} d y\right)^{\alpha / n}
$$

and then the corresponding $b$ and $g$ verify

$$
\begin{gathered}
\mu\left(\left\{(x, t) \in R_{+}^{n+1}:\|T b(x, t)\|_{B}>\gamma\right\}\right) \leq \frac{C}{\gamma^{s}}\left(\int_{R^{n}}\|f(y)\|_{A} d y\right)^{s}, \\
\|T g\|_{L_{B}^{\infty}(d \mu)} \leq C\|g\|_{L_{A}^{n / \alpha}(d x)} \leq C \lambda^{1-\alpha / n}\|f\|_{L_{A}^{1}(d x)}^{\alpha / n}=C \gamma .
\end{gathered}
$$

In other words

$$
\begin{aligned}
& \mu\left(\left\{(x, t) \in R_{+}^{n+1}:\|T f(x, t)\|_{B}>2 C \gamma\right\}\right) \\
& \leq \mu\left(\left\{(x, t) \in R_{+}^{n+1}:\|T b(x, t)\|_{B}>C \gamma\right\}\right) \\
& \leq \frac{C}{\gamma^{s}}\left(\int_{R^{n}}\|f(y)\|_{A} d y\right)^{s} .
\end{aligned}
$$


This finishes the proof of Theorem 1.

REMARK. If the hypothesis on the boundedness of $T$ in Theorem 1 is substituted by the following

(c) there exists $1 \leq p_{0}, q_{0}<\infty$ with $s / q_{0}<1$ and $1 / p_{0}=(\alpha / n)+\left(s / q_{0}\right)(1-\alpha / n)$ such that $T$ maps $L_{A}^{p_{0}}(d x)$ into $L_{B}^{q_{0}}(d \mu)$, then the following result can be proved:

THEOREM 5. If $T$ is an operator verifying (c) above and parts (a) and (b) of Theorem 1, then:

(i) $T$ maps $L_{A}^{p}(d x)$ into $L_{B}^{q}(d \mu)$ for $1 / p=\theta / p_{0}+(1-\theta), 1 / q=\theta / q_{0}+(1-\theta) / s$, $0<\theta \leq 1$ (i.e. $1 / p=\alpha / n+s(1-\alpha / n) / q, s<q<\infty)$.

(ii) $T$ maps $L_{A}^{1}(d x)$ into weak- $L_{B}^{s}(d \mu)$.

To see this observe that the proof of Theorem 1 can be reproduced here and with the same $\lambda$ we can obtain that

$$
\begin{aligned}
& \mu\left(\left\{(x, t) \in R_{+}^{n+1}:\|T g(x, t)\|_{B}>\gamma\right\}\right) \\
& \leq \frac{C}{\gamma^{q_{0}}} \int_{R_{+}^{n+1}}\|T g(x, t)\|_{B}^{q_{0}} d \mu(x, t) \leq \frac{C}{\gamma^{q_{0}}}\left(\int_{R^{n}}\|g(x)\|_{A}^{p_{0}} d x\right)^{q_{0} / p_{0}} \\
& \quad \leq C \frac{\lambda^{\left(p_{0}-1\right) q_{0} / p_{0}}}{\gamma^{q_{0}}}\left(\int_{R^{n}}\|f(x)\|_{A} d x\right)^{q_{0} / p_{0}} \leq \frac{C}{\gamma^{s}}\left(\int_{R^{n}}\|f(x)\|_{A} d x\right)^{s} .
\end{aligned}
$$

\section{Applications.}

THEOREM 6. Let $\alpha$ be given, $0 \leq \alpha<n$ and $0<1 / s \leq 1-\alpha / n$. Let $\varphi$ be a measurable function on $R^{n}$ such that:

(a) $|\varphi(x)| \leq C /(|x|+A)^{n+1-\alpha}$,

(b) $|\nabla \varphi(x)| \leq C /(|x|+B)^{n+2-\alpha}$,

where $A, B, C$ are constant independent of $x$.

Given the function $\Phi(x, t)=\varphi(x / t) / t^{n-\alpha}, t \geq 0$, consider the operator

$$
T f(x, t)=\int_{R^{n}} \Phi(x-y, t) f(y) d y .
$$

Then for any generalized Carleson measure $\mu$ on $R_{+}^{n+1}$ of order $s(1-\alpha / n), T$ is bounded from $L^{p}(d x)$ into $L^{q}(d \mu)$ for $1 / p=(\alpha / n)+(s / q)(1-\alpha / n), s<q<\infty$, and from $L^{1}(d x)$ into weak- $L^{s}(d \mu)$.

Moreover if $\alpha=0$ and $s=1$, the following vector-valued inequalities hold:

$$
\left\|\left(\sum_{j}\left|T f_{j}\right|^{q}\right)^{1 / q}\right\|_{L^{p}(d \mu)} \leq C\left\|\left(\sum_{j}\left|f_{j}\right|^{q}\right)^{1 / q}\right\|_{L^{p}(d x)} \quad(1<p, q<\infty)
$$

and

$\mu\left(\left\{(x, t) \in R_{+}^{n+1}: \sum_{j}\left|T f_{j}(x, t)\right|^{q}>\lambda^{q}\right\}\right) \leq \frac{C}{\lambda} \int_{R^{n}}\left(\sum_{j}\left|f_{j}(x)\right|^{q}\right)^{1 / q} d x$

$(1<q<\infty)$.

If we take $\varphi(x)=P(x)=c_{n}\left(|x|^{2}+1\right)^{-(n+1) / 2}$, then $\Phi(x, t)=P(x, t)$ and 
Theorem 6 gives

COROLlaRY 1. The Poisson integral verifies vector-valued inequalities (1) and $\left(1^{\prime}\right)$ for $1<p=q<\infty$ and $q=1$, respectively, $\mu$ being a Carleson measure and $1<r<\infty$.

PROOF OF THEOREM 6. Let us check that the operator $T$ satisfies the hypothesis of Theorem 1.

It is clear that $T$ maps $L^{n / \alpha}(d x)$ into $L^{\infty}(d \mu)$ since

$$
|T f(x, t)| \leq\|f\|_{L^{n / \alpha}(d x)}\|\varphi\|_{L^{(n / \alpha)^{\prime}(d x)}} .
$$

On the other hand, an advanced calculus computation shows that

$$
|\nabla \Phi(x, t)| \leq \frac{C}{(|x|+t)^{n+1-\alpha}}
$$

and then the kernel $K(x, y, t)=\Phi(x-y, t)$ of the operator $T$ verifies condition (b) of Theorem 1 since for $y, y^{\prime} \in R^{n}$

$$
\begin{aligned}
& \int_{\left|x-y^{\prime}\right|+t>2\left|y-y^{\prime}\right|}\left|K(x, y, t)-K\left(x, y^{\prime}, t\right)\right|^{s} d \mu(x, t) \\
& \quad \leq C \int_{\left|x-y^{\prime}\right|+t>2\left|y-y^{\prime}\right|} \frac{\left|y-y^{\prime}\right|^{s}}{\left(\left|x-y^{\prime}\right|+t\right)^{(n+1-\alpha) s}} d \mu(x, t)
\end{aligned}
$$

and if $Q$ is a cube in $R^{n}$ with center in $y^{\prime}$ and such that $y \in Q$, then the last expression is less than

$$
C|Q|^{s / n} \int_{\left|x-y^{\prime}\right|+t>2|Q|^{1 / n}} \frac{d \mu(x, t)}{\left(\left|x-y^{\prime}\right|+t\right)^{(n+1-\alpha) s}} .
$$

Now, the procedure in the last part of the proof of Theorem 3 can be repeated and so the above expression is less than

$$
|Q|^{s / n} \sum_{j} \frac{\mu\left(2^{j+1} Q\right)}{\left(2^{j}|Q|^{1 / n}\right)^{(n+1-\alpha) s}} \leq C \sum_{j} \frac{1}{2^{j s}} \frac{\mu\left(2^{j+1} Q\right)}{\left|2^{j+1} Q\right|^{(1-\alpha / n) s}} \leq C .
$$

Therefore the first assertions in Theorem 6 are obtained as a consequence of Theorem 1.

To finish the proof observe that in case $\alpha=0$ the inequalities (13) and (14) tell us that the hypotheses of Theorem 2 are fulfilled.

In our context the following theorem is parallel to a maximal theorem due to F. Zo (see [11]).

THEOREM 7. Let $\alpha$ be given, $0 \leq \alpha<n$ and $0<1 / s \leq 1-\alpha / n$. Let $\mu$ be a generalized Carleson measure on $R_{+}^{n+1}$ of order $s(1-\alpha / n)$ and $\varphi$ a measurable function in $R_{+}^{n+1}$ such that:

$$
\int_{R^{n}}|\varphi(x, t)|^{n /(n-\alpha)} d x \leq A<\infty \quad \forall t \geq 0
$$

(b) $\int_{\left|x-y^{\prime}\right|+t>2\left|y-y^{\prime}\right|} \sup _{\delta>0} \frac{1}{\delta^{n-\alpha}}\left|\varphi\left(\frac{x-y}{\delta}, \frac{t}{\delta}\right)-\varphi\left(\frac{x-y^{\prime}}{\delta}, \frac{t}{\delta}\right)\right|^{s} d \mu(x, t)<\infty$

for $y, y^{\prime} \in R^{n}$. 
Then the operator

$$
M_{\varphi} f(x, t)=\sup _{\delta>0}\left|\frac{1}{\delta^{n-\alpha}} \int_{R^{n}} \varphi\left(\frac{x-y}{\delta}, \frac{t}{\delta}\right) f(y) d y\right|
$$

is bounded from $L^{p}(d x)$ into $L^{q}(d \mu)$ for $1 / p=(\alpha / n)+(s / q)(1-\alpha / n), s<q<\infty$, and from $L^{1}(d x)$ into weak- $L^{s}(d \mu)$.

Moreover if $\alpha=0, s=1$ and $\varphi$ verifies

$\left(\mathrm{b}^{\prime}\right)|\nabla \varphi(x, t)| \leq C /(|x|+t)^{n+1}$,

then the following vector-valued inequalities hold:

$$
\left\|\left(\sum_{j}\left|M_{\varphi} f_{j}\right|^{q}\right)^{1 / q}\right\|_{L^{p}(d \mu)} \leq C\left\|\left(\sum_{j}\left|f_{j}\right|^{q}\right)^{1 / q}\right\|_{L^{p}(d x)} \quad(1<p, q<\infty)
$$

and

$\mu\left(\left\{(x, t) \in R_{+}^{n+1}: \sum_{j}\left|M_{\varphi} f_{j}(x, t)\right|^{q}>\lambda^{q}\right\}\right) \leq \frac{C}{\lambda} \int_{R^{n}}\left(\sum_{j}\left|f_{j}(x)\right|^{q}\right)^{1 / q} d x$

$(1<q<\infty)$.

Before proving Theorem 7 we state and prove two corollaries.

COROLLARY 2. The maximal operator $\mathcal{M}$ defined in the introduction verifies inequalities (1) and ( $\left.1^{\prime}\right)$ for $1<p=q<\infty$ and $q=1$, respectively, $\mu$ being $a$ Carleson measure and $1<r<\infty$.

COROllary 3. For $0<\alpha<n$ and $0<1 / s \leq 1-\alpha / n$, the maximal operator $\mathcal{M}_{\alpha}$ is bounded from $L^{p}(d x)$ into $L^{q}(d \mu)$ for $1 / p=(\alpha / n)+(s / q)(1-\alpha / n), s<q<$ $\infty$, and from $L^{1}(d x)$ into weak- $L^{s}(d \mu), \mu$ being a generalized Carleson measure of order $s(1-\alpha / n)$.

The proof of both corollaries is the same and consists in taking a function $\varphi_{\alpha}$ in $R^{n+1}$ such that if $Q_{0}$ is the unit cube in $R^{n+1}$, then

$$
\chi_{Q_{0}} \leq \varphi_{\alpha} \leq \chi_{2 Q_{0}}
$$

and

$$
\left|\nabla \varphi_{\alpha}(y)\right| \leq C|y|^{\alpha-n-1} \quad \text { for all } y \in R^{n+1} \backslash\{0\}
$$

(in case $0<\alpha<n$, it is easy to see that this fact implies condition (b) of Theorem 7 for $\left.\varphi_{\alpha}\right)$. Now, observe that $\mathcal{M}_{\alpha} f(x, t) \leq M_{\varphi_{\alpha}} f(x, t)$ for $(x, t) \in R_{+}^{n+1}$ and apply Theorem 7.

PROOF OF THEOREM 7. Let $S$ be the linear operator defined by

$$
S f(x, t)=\left\{\frac{1}{\delta^{n-\alpha}} \int_{R^{n}} \varphi\left(\frac{x-y}{\delta}, \frac{t}{\delta}\right) f(y) d y\right\}_{\delta>0} .
$$

By (a) it is clear that $S$ is bounded from $L^{n / \alpha}(d x)$ into $L_{l \infty}^{\infty}(d \mu)$, moreover $S$ is given by a $\mathcal{L}\left(\mathbf{C}, l^{\infty}\right) \cong l^{\infty}$-valued kernel

$$
K(x, y, t)=\left\{\frac{1}{\delta^{n-\alpha}} \varphi\left(\frac{x-y}{\delta}, \frac{t}{\delta}\right)\right\}_{\delta>0}
$$


Condition (b) says that Theorem 1 can be applied to $S$. Moreover, condition $\left(\mathrm{b}^{\prime}\right)$ says that for $\left|x-y^{\prime}\right|+t>2\left|y-y^{\prime}\right|$

$$
\begin{aligned}
\frac{1}{\delta^{n}}\left|\varphi\left(\frac{x-y}{\delta}, \frac{t}{\delta}\right)-\varphi\left(\frac{x-y^{\prime}}{\delta}, \frac{t}{\delta}\right)\right| & \leq \frac{C}{\delta^{n}} \frac{\left|y-y^{\prime}\right| \delta^{-1}}{\left(\left|x-y^{\prime}\right| \delta^{-1}+t \delta^{-1}\right)^{n+1}} \\
& \leq C \frac{\left|y-y^{\prime}\right|}{\left(\left|x-y^{\prime}\right|+t\right)^{n+1}} .
\end{aligned}
$$

In particular this means that

$$
\left\|K(x, y, t)-K\left(x, y^{\prime}, t\right)\right\|_{l \infty} \leq C \frac{\left|y-y^{\prime}\right|}{\left(\left|x-y^{\prime}\right|+t\right)^{n+1}} \quad \text { for }\left|x-y^{\prime}\right|+t>2\left|y-y^{\prime}\right| .
$$

So Theorem 2 applies to $S$.

The proof is finished by observing that $M_{\varphi} f=\|S f\|_{l^{\infty}}$.

The results in Corollary 3 can be improved, in fact a vector-valued version is true. This will be a consequence of the following

Proposition. Let $0<\alpha<n$ and $0<1 / s \leq 1-\alpha / n$. Let $\mu$ be a generalized Carleson measure of order $s(1-\alpha / n)$ and $r, 1 \leq r \leq \infty$. Then the generalized fractional integral of order $\alpha, T_{\alpha}$, defined in the introduction verifies the following inequalities:

(i)

$$
\begin{gathered}
\left\{\int_{R_{+}^{n+1}}\left(\sum_{j}\left|T_{\alpha} f_{j}(x, t)\right|^{r}\right)^{q / r} d \mu(x, t)\right\}^{1 / q} \\
\leq C\left\{\int_{R^{n}}\left(\sum_{j}\left|f_{j}(x)\right|^{r}\right)^{p / r} d x\right\}^{1 / p}
\end{gathered}
$$

for $1 / p=(\alpha / n)+(s / q)(1-\alpha / n), s<q<\infty$.

(ii)

$$
\begin{gathered}
\mu\left(\left\{(x, t) \in R_{+}^{n+1}: \sum_{j}\left|T_{\alpha} f_{j}(x, t)\right|^{r}>\lambda^{r}\right\}\right) \\
\leq \frac{C}{\lambda^{s}}\left\{\int_{R^{n}}\left(\sum_{j}\left|f_{j}(x)\right|^{r}\right)^{1 / r} d x\right\}^{s} .
\end{gathered}
$$

COROLlARY 4. The same inequalities are true for the maximal operator $\mathcal{M}_{\alpha}$.

For the proof of the corollary observe that

$$
\mathcal{M}_{\alpha} f(x, t) \leq C \cdot T_{\alpha} f(x, t), \quad(x, t) \in R_{+}^{n+1} .
$$

Proof of The Proposition. The idea is to check that Theorem 5 can be applied. This will be true because of the following lemma. 
LEMmA. Let $\alpha$ be given, $0<\alpha<n$. Then for any $\varepsilon>0$ with $0<\alpha-\varepsilon<$ $\alpha+\varepsilon<n$ there exists a constant $C_{\varepsilon}$ such that

$$
T_{\alpha} f(x, t) \leq C_{\varepsilon}\left(\mathcal{M}_{\alpha+\varepsilon} f(x, t) \cdot \mathcal{M}_{\alpha-\varepsilon} f(x, t)\right)^{1 / 2}, \quad(x, t) \in R_{+}^{n+1} .
$$

Taking the lemma for granted and verifying $p_{0}$ and $q_{0}$ :

$$
1 / p_{0}=(\alpha / n)+\left(s / q_{0}\right)(1-\alpha / n), \quad s<q_{0}<\infty,
$$

choose $q_{\varepsilon}$ and $\bar{q}_{\varepsilon}$ given by

$$
q_{\varepsilon}\left(1-\frac{\alpha-\varepsilon}{n}\right)=q_{0}\left(1-\frac{\alpha}{n}\right), \quad \bar{q}_{\varepsilon}\left(1-\frac{\alpha+\varepsilon}{n}\right)=q_{0}\left(1-\frac{\alpha}{n}\right) .
$$

Observe that

$$
q_{\varepsilon}<q_{0}<\bar{q}_{\varepsilon} \text { and }\left(q_{0} / 2 q_{\varepsilon}\right)+\left(q_{0} / 2 \bar{q}_{\varepsilon}\right)=1 .
$$

Then Hölder's inequality says that

$$
\begin{aligned}
\int_{R_{+}^{n+1}}\left|T_{\alpha} f(x, t)\right|^{q_{0}} d \mu(x, t) \\
\leq C_{\varepsilon} \int_{R_{+}^{n+1}}\left|\mathcal{M}_{\alpha+\varepsilon} f(x, t) \cdot \mathcal{M}_{\alpha-\varepsilon} f(x, t)\right|^{q_{0} / 2} d \mu(x, t) \\
\leq C_{\varepsilon}\left(\int_{R_{+}^{n+1}} \mathcal{M}_{\alpha+\varepsilon} f(x, t)^{\bar{q}_{\varepsilon}} d \mu(x, t)\right)^{q_{0} / 2 \bar{q}_{\varepsilon}} \\
\\
\cdot\left(\int_{R_{+}^{n+1}} \mathcal{M}_{\alpha-\varepsilon} f(x, t)^{q_{\varepsilon}} d \mu(x, t)\right)^{q_{0} / 2 q_{\varepsilon}}
\end{aligned}
$$

and by Corollary 3 this is less than

$$
C_{\varepsilon}\left(\int_{R^{n}}|f(x)|^{p_{0}} d x\right)^{q_{0} / 2 p_{0}}\left(\int_{R^{n}}|f(x)|^{p_{0}} d x\right)^{q_{0} / 2 p_{0}}=C_{\varepsilon}\|f\|_{L^{p_{0}}(d x)}^{q_{0}} .
$$

On the other hand the computations in the proof of Theorem 6 can be reproduced to see that the kernel of $T_{\alpha}$ satisfies condition (b) of Theorem 5 .

Therefore $T_{\alpha}$ maps $L^{p}(d x)$ into $L^{q}(d \mu)$ for $1 / p=(\alpha / n)+(s / q)(1-\alpha / n), s<$ $q<\infty$, and $L^{1}(d x)$ into weak- $L^{s}(d \mu)$.

To obtain the required vector-valued inequalities it is a well-known fact that if $(\Omega, A, m)$ is an arbitrary measure space, $B$ is a Banach space and $T$ is a positive linear operator acting on measurable functions (i.e. if $f \geq 0$, then $T f \geq 0$ ), then for any nice $B$-valued function $f$, let us say $f \in B \otimes L^{\bar{p}}(\Omega, A, m)$, the following pointwise inequality is true:

$$
\|T f(x)\|_{B} \leq T\left(\|f\|_{B}\right)(x), \quad x \in \Omega .
$$

Taking $T=T_{\alpha}$ and $B=l^{r}$ in (17), one obtains the vector-valued inequalities stated in the proposition.

ProOf OF THE LEMMA. We follow [10 and 2]. Given $(x, t) \in R_{+}^{n+1}$ and $\varepsilon>0$, $0<\alpha-\varepsilon<\alpha+\varepsilon<n$, we choose $\delta$ such that

$$
\delta^{2 \varepsilon}=\mathcal{M}_{\alpha+\varepsilon} f(x, t) \cdot\left(\mathcal{M}_{\alpha-\varepsilon} f(x, t)\right)^{-1} .
$$


Now we put

$$
\begin{aligned}
T_{\alpha} f(x, t)= & c_{n} \int_{|x-y|+t<\delta} \frac{f(y)}{(|x-y|+t)^{n-\alpha}} d y \\
& +c_{n} \int_{|x-y|+t>\delta} \frac{f(y)}{(|x-y|+t)^{n-\alpha}} d y=\mathrm{I}_{1}+\mathrm{I}_{2} .
\end{aligned}
$$

Let $R_{i}$ and $B_{i}(i \in Z)$ be the sets

$$
\begin{aligned}
& R_{i}=\left\{y \in R^{n}: 2^{-i-1} \delta<|x-y|+t<2^{-i} \delta\right\} \\
& B_{i}=\left\{y \in R^{n}:|x-y|<2^{-i} \delta\right\} .
\end{aligned}
$$

Observe that if $t>\delta$, then $\mathrm{I}_{1}=0$ and in any case

$$
\begin{aligned}
\left|\mathrm{I}_{1}\right| & \leq C \sum_{i=0}^{\infty} \int_{R_{i}}\left(2^{-i-1} \delta\right)^{-n+\alpha}|f(y)| d y \\
& \leq C \sum_{i=0}^{\infty} \frac{1}{\left(2^{-i} \delta\right)^{n-\alpha}} \int_{B_{i}}|f(y)| d y \\
& \leq C \sum_{i=0}^{\infty}\left(2^{-i} \delta\right)^{\varepsilon} \frac{1}{\left(2^{-i} \delta\right)^{n-\alpha+\varepsilon}} \int_{B_{i}}|f(y)| d y \\
& \leq C_{\varepsilon} \delta^{\varepsilon} \mathcal{M}_{\alpha-\varepsilon} f(x, t) .
\end{aligned}
$$

Analogously

$$
\begin{aligned}
\left|\mathrm{I}_{2}\right| & \leq C \sum_{i=1}^{\infty}\left(2^{i} \delta\right)^{-\varepsilon} \frac{1}{\left(2^{i} \delta\right)^{n-\alpha-\varepsilon}} \int_{B_{-i}}|f(y)| d y \\
& \leq C_{\varepsilon}^{\prime} \delta^{-\varepsilon} \mathcal{M}_{\alpha+\varepsilon} f(x, t),
\end{aligned}
$$

and so with the above election of $\delta$ the lemma is proved.

REMARKS. 1. The Poisson integral is a linear positive operator. Then any vector-valued extension can be obtained from (17).

2. The linearity of the operator is essential in (17). This can be released with the following example:

Take the sequence of functions $f_{j}=\chi_{\left[2^{-j-1,2-j]}\right.}$ on $R$. Let $T$ be the standard Hardy-Littlewood maximal operator and let $q, 1 \leq q<\infty$, be given. For $B=l^{q}$ inequality (17) means

$$
T\left[\left(\sum_{j=0}^{\infty}\left|f_{j}\right|^{q}\right)^{1 / q}\right](x) \geq\left(\sum_{j=0}^{\infty}\left|T f_{j}(x)\right|^{q}\right)^{1 / q}
$$

and this is

$$
T\left(\chi_{[0,1]}\right)(x) \geq\left(\sum_{j=0}^{\infty}\left|T f_{j}(x)\right|^{q}\right)^{1 / q}
$$

That is false in $x=0$; moreover when $x$ increases to zero the left-hand side remains bounded while the right-hand side tends to infinity.

3. The translation invariance of this operator is the first variable that makes it possible to obtain mixed norm estimates in some particular case. This will be 
considered in a forthcoming paper but we can state the following example of mixed norms: true:

If $\mu$ is a Carleson measure on $R_{+}^{2}$, then the following mixed norm estimate is

$$
\begin{aligned}
& \left(\int\left(\int \ldots \int\left(\int\left|\mathcal{M} f\left(x_{1}, \ldots, x_{n}, t\right)\right|^{p_{1}} d x_{1}\right)^{p_{2} / p_{1}} d x_{2} \cdots\right)^{p_{n} / p_{n-1}} d \mu\left(x_{n}, t\right)\right)^{1 / p_{n}} \\
& \leq C\left(\int\left(\int \ldots \int\left(\int\left|f\left(x_{1}, \ldots, x_{n}\right)\right|^{p_{1}} d x_{1}\right)^{p_{2} / p_{1}} d x_{2} \cdots\right)^{p_{n} / p_{n-1}} d x_{n}\right)^{1 / p_{n}}
\end{aligned}
$$

$1<p_{i}<\infty, i=1, \ldots, n$.

5. Some weighted norm inequalities. The main theorem in this part is the following

THEOREM 8. Let $p, q$ be given, $1 \leq p \leq q<\infty, 0 \leq \alpha<n$. Then the following are equivalent:

(i) The operator $\mathcal{M}_{\alpha}$ is bounded from $L^{p}(d \omega)$ into weak- $L^{q}(d \mu)$.

(ii) The pair $(\mu, \omega)$ satisfies condition $C_{q, p, \alpha}$ (see §2).

The idea of the proof we shall give is taken from the proof of the corresponding dyadic theorem; in order to state it we define

$$
\mathcal{N}_{\alpha} f(x, t)=\sup _{Q}\left\{\frac{1}{|Q|^{1-\alpha / n}} \int_{Q}|f(y)| d y\right\} \quad\left(x \in R^{n}, t \geq 0\right),
$$

where the supremum is taken over the dyadic cubes $Q$ (i.e. cubes of the form $\prod_{i=1}^{n}\left[x_{i}, x_{i}+2^{k}\right)$, where $\left.x \in 2^{k} Z^{n}, k \in Z\right)$ in $R^{n}$ containing $x$ and have side length at least $t$.

We shall say that the pair $(\mu, \omega)$ satisfies dyadic $C_{q, p, \alpha}$ if condition (2) is only fulfilled for dyadic cubes $Q$ in $R^{n}$.

DYADIC TheOREM 8. Let $p, q$ be given, $1 \leq p \leq q<\infty, 0<\alpha<n$. Then the following are equivalent:

(i) The operator $\mathcal{N}_{\alpha}$ is bounded from $L^{p}(d \omega)$ into weak- $L^{q}(d \mu)$.

(ii) The pair $(\mu, \omega)$ satisfies dyadic $C_{q, p, \alpha}$.

The proof of (i) $\Rightarrow$ (ii) is the same in both theorems; we write it for the dyadic case.

It is clear that

$$
\tilde{Q} \subset\left\{(x, t) \in R_{+}^{n+1}: \mathcal{N}_{\alpha} f(x, t) \geq \frac{1}{|Q|^{1-\alpha / n}} \int_{Q}|f(y)| d y\right\} .
$$

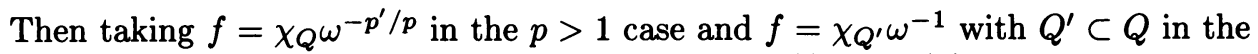
$p=1$ case (let $Q^{\prime}$ tend to $x$ ), the use of hypothesis (i) gives (ii).

PROOF OF (ii) $\Rightarrow$ (i). Dyadic case. It is enough to prove that $\mathcal{N}_{\alpha}^{R}$ is bounded from $L^{p}(d \omega)$ into weak- $L^{q}(d \mu)$ with bound independent of $R$ and $\mathcal{N}_{\alpha}^{R}$ the operator defined by

$$
\mathcal{N}_{\alpha}^{R} f(x, t)=\sup _{Q}\left\{\frac{1}{|Q|^{1-\alpha / n}} \int_{Q}|f(y)| d y\right\} \quad\left(x \in R^{n}, t \geq 0\right)
$$


where the supremum is taken over the dyadic cubes in $R^{n}$ containing $x$ and have side length bigger than $t$ but smaller than $R$.

The bound $R$ for the side length of the cubes permits us to make a standard maximality argument and prove that for each $\lambda>0$ there exists a family $\left\{Q_{j}\right\}$ of dyadic cubes in $R^{n}$ such that

$$
E_{\lambda}=\left\{(x, t) \in R_{+}^{n+1}: \mathcal{N}_{\alpha}^{R} f(x, t)>\lambda\right\}=\bigcup \tilde{Q}_{j},
$$

The interiors of $\tilde{Q}_{j}$ are disjoints,

$$
\frac{1}{\left|Q_{j}\right|^{1-\alpha / n}} \int_{Q_{j}}|f(y)| d y>\lambda \text {. }
$$

Then

$$
\begin{aligned}
\mu\left(\left\{(x, t) \in R_{+}^{n+1}: \mathcal{N}_{\alpha}^{R} f(x, t)>\lambda\right\}\right) & =\sum_{j} \mu\left(\tilde{Q}_{j}\right) \\
& \leq \sum_{j} \frac{\mu\left(\tilde{Q}_{j}\right)}{\lambda^{q}} \cdot \frac{1}{\left|Q_{j}\right|^{(1-\alpha / n) q}}\left(\int_{Q_{j}}|f(y)| d y\right)^{q} .
\end{aligned}
$$

In the $p=1$ case the condition $C_{q, 1, \alpha}$ gives directly that this is less than

$$
\frac{C}{\lambda^{q}} \sum_{j}\left(\int_{Q_{j}}|f(y)| \omega(y) d y\right)^{q} \leq \frac{C}{\lambda^{q}}\left(\int_{R^{n}}|f(y)| \omega(y) d y\right)^{q} .
$$

In the other case, $p>1$, Hölder's inequality says that $\mu\left(E_{\lambda}\right)$ is less than

$$
\begin{aligned}
& \frac{1}{\lambda^{q}} \sum_{j} \frac{\mu\left(\tilde{Q}_{j}\right)}{\left|Q_{j}\right|^{(1-\alpha / n) q}}\left(\int_{Q_{j}}|f(y)|^{p} \omega(y) d y\right)^{q / p}\left(\int_{Q_{j}} \omega^{-p^{\prime} / p} d y\right)^{q / p^{\prime}} \\
& \leq \frac{C}{\lambda^{q}}\left(\int_{R^{n}}|f(x)|^{p} \omega(x) d x\right)^{q / p}
\end{aligned}
$$

where condition $C_{q, p, \alpha}$ and the fact $p \leq q$ have been used.

ProOF OF (ii) $\Rightarrow$ (i). Nondyadic case. The proof can be done as in the dyadic case if we prove the following

LEMMA. Given $0<\lambda<\infty$, there exists a sequence of cubes $\left\{Q_{k}\right\}$ in $R^{n}$ such that :

(i)

$$
\left\{(x, t) \in R_{+}^{n+1}: \mathcal{M}_{\alpha}^{R} f(x, t)>\lambda\right\} \subset \bigcup_{k} \tilde{Q}_{k}
$$

(ii)

$$
\frac{1}{\left|Q_{k}\right|^{1-\alpha / n}} \int_{Q_{k}}|f(y)| d y>c \lambda
$$

(where $c$ is a positive constant depending only on $\alpha$ and $n$ ).

(iii) The sequence $\left\{\tilde{Q}_{k}\right\}$ can be distributed in $N$ (number depending on the dimension $n$ ) families of cubes with disjoint interiors.

(Here, $\mathcal{M}_{\alpha}^{R}$ has obvious meaning.) 
PROOF OF THE LEMMA. Let $\bar{M}_{\alpha} f(x, t)$ be the noncentered maximal function, i.e. the supremum in the definition of $\mathcal{M}_{\alpha}$ is taken over all the cubes containing $x$. Analogously we define $\bar{M}_{\alpha}^{R}$.

The existence of constants $c_{n}$ and $k_{n}$ independent of $R$ and bigger than 1 is clear, such that

$$
\mathcal{M}_{\alpha}^{R} f(x, t) \leq \bar{M}_{\alpha}^{R} f(x, t) \leq c_{n} \mathcal{M}_{\alpha}^{k_{n} R} f(x, t), \quad(x, t) \in R_{+}^{n+1} .
$$

Let $f_{\alpha}^{* R}(x)$ be the standard centered fractional maximal operator truncated at $R$, i.e.

$$
f_{\alpha}^{* R}(x)=\sup _{Q}\left\{\frac{1}{|Q|^{1-\alpha / n}} \int_{Q}|f(y)| d y\right\}, \quad x \in R^{n},
$$

where the supremum is taken over cubes in $R^{n}$ centered at $x$, with sides parallel to the axes and have side length less than $R$.

We define the following sets:

$$
\begin{aligned}
& E_{\eta}^{R}=\left\{(x, t) \in R_{+}^{n+1}: \mathcal{M}_{\alpha}^{R} f(x, t)>\eta\right\} \\
& A_{\eta}^{R}=\left\{x \in R^{n}: f_{\alpha}^{* R}(x)>\eta\right\}
\end{aligned}
$$

For $x \in A_{\eta}^{R}$ let $t(x, \eta, R)=\sup \left\{t:(x, t) \in E_{\eta}^{R}\right\}$; then the following statements are obvious:

(a) $t(x, \eta, R) \leq R ; x \in R^{n}, \eta>0$.

(b) If $Q(x, \eta, R)$ is the cube centered at $x$ and with radius $t(x, \eta, R)$, then

$$
\frac{1}{|Q(x, \eta, R)|^{1-\alpha / n}} \int_{Q(x, \eta, R)}|f(y)| d y>\eta \text {. }
$$

(c) $A_{\eta}^{R} \subset \bigcup_{x \in A_{\eta}^{R}} Q(x, \eta, R)$.

A standard application of Besicovitch's covering lemma says that there exists a sequence $\left\{x_{k}\right\}$ such that

$$
A_{\eta}^{R} \subset \bigcup_{k} Q\left(x_{k}, \eta, R\right)=\bigcup_{k} Q_{k}
$$

and the family $\left\{Q_{k}\right\}$ can be distributed in $N$ disjoint subfamilies ( $N$ depending only on $n$ ).

Then the lemma will be proved if we show

$$
E_{\lambda}^{R} \subset \bigcup_{k} \tilde{Q}\left(x_{k}, c_{n}^{-1} \lambda, k_{n} R\right)
$$

Let $(x, t) \in E_{\lambda}^{R} \subset E_{c_{n}^{-1} \lambda}^{k_{n} R}$; in particular

$$
x \in A_{c_{n}^{-1} \lambda}^{k_{n} R} \subset \bigcup_{k} Q\left(x_{k}, c_{n}^{-1} \lambda, k_{n} R\right)
$$

and then there exists a $k$ such that

$$
x \in Q\left(x_{k}, c_{n}^{-1} \lambda, k_{n} R\right) .
$$


If $(x, t) \notin Q\left(x_{k}, c_{n}^{-1} \lambda, k_{n} R\right)$, then $t>t\left(x_{k}, c_{n}^{-1} \lambda, k_{n} R\right)$ and this would say that $x_{k}$ is in any cube, $Q(x ; s)$, centered at $x$ and with radius $s \geq t$, therefore

$$
\mathcal{M}_{\alpha}^{R} f(x, t)=\overline{\mathcal{M}}_{\alpha}^{R} f\left(x_{k}, t\right)
$$

Now using (22) and the fact that $(x, t) \in E_{\lambda}^{R}$ we have

$$
\lambda<c_{n} \mathcal{M}_{\alpha}^{k_{n} R} f\left(x_{k}, t\right),
$$

which is a contradiction to the definition of $t\left(x_{k}, c_{n}^{-1} \lambda, k_{n} R\right)$.

REMARKS. 1. The case $\alpha=0$ has already been considered in [7] where a different proof was given.

2. In order to prove that conditions $C_{q, p, \alpha}$ ad $C_{q, 1, \alpha}$ are necessary we have assumed that $\omega^{-p^{\prime} / p}$ and respectively $\omega^{-1}$ are locally integrable; this can be done because in the other case (i) fails; in fact: If $\int_{Q} \omega^{-p^{\prime} / p}(x) d x=\infty$, then $\int_{Q} \omega^{1-p^{\prime}}(x) d x$ $=\int_{Q} \omega^{-p^{\prime}}(x) \omega(x) d x=\infty$ and this will imply the existence of $g \in L^{p}(Q ; \omega(x) d x) \subset$ $L^{p}(\omega(x) d x)$ such that $\int_{Q} g(x) \omega^{-1}(x) \omega(x) d x=\infty$ and then $\mathcal{M}_{\alpha} g(x, t)=\infty$ for $(x, t) \in R_{+}^{n+1}$.

If $\int_{Q} \omega^{-1}(x) d x=\infty$ this will say that $\mathcal{M}_{\alpha}\left(\chi_{Q} \omega^{-1}\right) \equiv \infty$ and $\chi_{Q} \omega^{-1} \in L^{1}(d \omega)$ which is a contradiction to (i)

3. If $t=0$, then $T_{\alpha} f(x, 0)$ and $\mathcal{M}_{\alpha} f(x, 0)$ are respectively the usual fractional integral $I_{\alpha} f$ and the maximal fractional operator $f_{\alpha}^{*}$ in $R^{n}$. In this case taking $d \mu(x, t)=u(x) d x \otimes \delta_{0}(t)$ we have some necessary and sufficient condition in the pair of weights $(u, \omega)$ for the weak boundedness of the operator $f_{\alpha}^{*}$. The case in which $(1 / q)=(1 / p)-(\alpha / n)$ and $u^{1 / q}=\omega^{1 / p}$ has been treated in [4]. The case in which $p=q$ for different weights and strong boundedness has been considered in [9].

4. The maximal operator $\mathcal{M}_{\alpha}$ can be defined with respect to any doubling measure $\sigma$ on $R^{n}$, i.e.

$$
\mathcal{M}_{\alpha} f(x, t)=\sup _{Q}\left\{\frac{1}{\sigma(Q)} \int_{Q}|f(y)| d \sigma(y)\right\}
$$

and, as Besicovitch's lemma remains valid, all the results in $\S 5$ are true substituting the Lebesgue measure for the measure $\sigma$.

In particular, for $\alpha=0, \omega \equiv 1$ and $\sigma$ doubling, Theorem 8 gives as a consequence the following well-known lemma about Carleson measures:

LEMMA 1. Let $\mu$ and $\sigma$ be nonnegative measures on $R_{+}^{n+1}$ and $R^{n}$ respectively such that $\sigma$ is doubling and $\mu(\tilde{Q}) \leq C \sigma(Q)^{q / p}$ for $q \geq p$. Then

$$
\mu\left(\left\{(x, t) \in R_{+}^{n+1}:|f(x, t)|>\lambda\right\}\right) \leq \frac{C}{\lambda^{q}}\left(\int_{R^{n}} N(f)(x)^{p} d \sigma(x)\right)^{q / p} .
$$

Here $N(f)(x)$ is the nontangential maximal function

$$
N(f)(x)=\sup _{(z, t) \in \Gamma(x)}|f(z, t)|,
$$

where $\Gamma(x)$ is the cone $\left\{(z, t) \in R_{+}^{n+1}:|x-z|<a t\right\}$ with vertex $x$ and aperture $a$.

The proof of the lemma is given by the inequality

$$
|f(x, t)| \leq C \mathcal{M}(N f)(x, t), \quad(x, t) \in R_{+}^{n+1} .
$$

This inequality allows us to state the following generalization of Lemma 1: 
LEMMA 2. Let $\mu, \nu, \sigma$ be nonnegative measures on $R_{+}^{n+1}, R^{n}$ and $R^{n}$ respectively such that $\sigma$ is doubling, $\nu$ is absolutely continuous with respect to $\sigma$ and such that for any cube $Q$ in $R^{n}$

$$
\mu(Q)\left(\frac{1}{\sigma(Q)} \int_{Q}\left(\frac{d \nu(x)}{d \sigma}\right)^{1-p^{\prime}} d \sigma(x)\right)^{q / p^{\prime}} \leq C \sigma(Q)^{q / p}
$$

Then

$$
\mu\left(\left\{(x, t) \in R_{+}^{n+1}:|f(x, t)|>\lambda\right\}\right) \leq \frac{C}{\lambda^{q}}\left(\int_{R^{n}}(N(f)(x))^{p} d \nu(x)\right)^{q / p} .
$$

5. The lemma stated in $\S 4$ allows us to obtain some weighted norm inequalities for the operator $T_{\alpha}$ and then by inequality (17) some vector-valued inequalities with weights.

In our opinion the main applications of this kind of inequality would be in the theory of Besov-Lizorkin-Triebel spaces (see [3]); this will be considered in a forthcoming paper.

\section{REFERENCES}

1. C. Fefferman and E. M. Stein, Some maximal inequalities, Amer. J. Math. 93 (1971), 107-115.

2. H. L. Hedberg, On certain convolution inequalities, Proc. Amer. Math. Soc. 36 (1972), 505-510.

3. H. P. Heinig and R. Johnson, Weighted norm inequalities for $L^{r}$-valued integral operators and applications, Math. Nachr. 107 (1982), 161-174.

4. B. Muckenhoupt and R. L. Wheeden, Weighted norm inequalities for fractional integrals, Trans. Amer. Math. Soc. 192 (1974), 261-274.

5. J. L. Rubio de Francia, Weighted norm inequalities and vector valued inequalities, Lecture Notes in Math., vol. 908, Springer-Verlag, Berlin, 1982, pp. 86-101.

6. J. L. Rubio de Francia, F. J. Ruiz and J. L. Torrea, Calderón-Zygmund theory for operatorvalued kernels, Advances in Math. (to appear).

7. F. J. Ruiz, A unified approach to Carleson measures and $A_{p}$ weights, Pacific J. Math. 117 (1985), 397-404.

8. F. J. Ruiz and J. L. Torrea, $A$ unified approach to Carleson measures and $A_{p}$ weights. II, Pacific J. Math. 120 (1985), 189-197.

9. E. T. Sawyer, A characterization of a two-weight norm inequality for maximal operators, Studia Math. 75 (1982), 1-11.

10. G. V. Welland, Weighted norm inequalities for fractional integrals, Proc. Amer. Math. Soc. 51 (1975), 143-148.

11. F. Zo, A note on approximation of the identity, Studia Math. 55 (1976), 11-122.

12. L. Hormander, Estimates for translation invariant operators in $L_{p}$ spaces, Acta Math. 104 (1960), 93-140.

13. J. Schwartz, A remark on inequalities of Calderón-Zygmund type for vector valued functions, Comm. Pure Appl. Math. 14 (1961), 785-799.

Departamento Teoría de funciones, facultad de Ciencias, Universidad de ZARAGOZA, ZARAGOZA, SPAIN

Departamento Matematicas, Facultad de Ciencias, Universidad Autónoma DE MADRID, MADRID, SPAIN 